\title{
DETERMINAÇÃO DE PARÂMETROS CINÉTICOS DE CURA DO COMPÓSITO DE EPÓXI REFORÇADO COM 20\% DE FIBRAS DE MALVA*
}

\section{Resumo}

Lucio Fabio Cassiano Nascimento ${ }^{1}$ Luis Henrique Leme Louro ${ }^{2}$ Sérgio Neves Monteiro Jheison Lopes dos Santos ${ }^{4}$ Édio Pereira Lima Júnior ${ }^{5}$ Fernanda Santos da $L u z^{6}$

A determinação dos parâmetros cinéticos de cura de uma resina polimérica, bem como do compósito de matriz polimérica constitui importante ferramenta científica e tecnológica para processos industriais. No presente trabalho foi utilizada a técnica de calorimetria diferencial de varredura (DSC), prevista pela ASTM E698, com taxas de $5,7,5$ e $10^{\circ} \mathrm{C} / \mathrm{min}$ para determinação da energia de ativação "E" ( $\left.\mathrm{kJ} / \mathrm{mol}\right)$, fator pré-exponencial " $\mathrm{Z}$ " $\left(\mathrm{min}^{-1}\right)$ e o tempo médio para alcançar $50 \%$ de cura " $\mathrm{t}_{1 / 2}$ " para 0 epóxi puro e para o compósito reforçado por fibras de malva, em temperaturas variando na faixa de $25^{\circ} \mathrm{C}$ a $100^{\circ} \mathrm{C}$. Os resultados mostraram que o tempo médio de cura de $24 \mathrm{~h}$, utilizado para a produção de placas de resina epóxi DGEBA-TETA ou compósitos de epóxi reforçados com fibras naturais, pode ser reduzido consideravelmente.

Palavras-chave: Cinética de cura; Calorimetria diferencial de varredura; Resina epóxi; Compósito reforçado por fibras de malva.

\section{DETERMINATION OF KINETIC PARAMETERS OF EPOXY COMPOSITE REINFORCED WITH $20 \%$ VOL. OF MALVA FIBERS}

\section{Abstract}

The determination of the curing kinetic parameters of a polymer resin as well as the polymer matrix composite constitutes an important scientific and technological tool for industrial processes. In the present work, differential scanning calorimetry (DSC), predicted by ASTM E698, with rates of $5,7.5$ and $10^{\circ} \mathrm{C} / \mathrm{min}$ was used to determine the activation energy "E" ( $\mathrm{kJ} / \mathrm{mol})$, Pre-exponential factor "Z" $\left(\mathrm{min}^{-1}\right)$ and the mean time to reach $50 \%$ "t $1 / 2$ " cure for the pure epoxy and the composite reinforced with malva fibers at temperatures ranging from $25^{\circ} \mathrm{C}$ to $100^{\circ} \mathrm{C}$. The results showed that the average curing time of 24 hours, used for the production of DGEBA-TETA epoxy resins or epoxy composites reinforced with natural fibers, can be considerably reduced.

Keywords: Cure kinetics; Differential scanning calorimetry; Epoxy resin; Composite reinforced by malva fibers.

1 Engenheiro Metalurgista, D.C, Professor Titular, Seção de Engenharia Mecânica e de Materiais, Instituto Militar de Engenharia, Rio de Janeiro, RJ, Brasil.

lucio_coppe@yahoo.com.br.

2 Engenheiro Metalurgista, Ph.D, Professor Titular, Seção de Engenharia Mecânica e de Materiais, Instituto Militar de Engenharia, Rio de Janeiro, RJ, Brasil.

3 Engenheiro Metalurgista, Ph.D, Professor Titular, Seção de Engenharia Mecânica e de Materiais, Instituto Militar de Engenharia, Rio de Janeiro, RJ, Brasil.

4 Físico, D.C, Bolsista Pós-doutorado, Seção de Engenharia Mecânica e de Materiais, Instituto Militar de Engenharia, Rio de Janeiro, RJ, Brasil.

5 Engenheiro Mecânico, M.C, Doutorando em Ciência dos Materiais, Seção de Engenharia Mecânica e de Materiais, Instituto Militar de Engenharia, Rio de Janeiro, RJ, Brasil.

6 Engenheira Metalurgista, M.C, Doutoranda em Ciência dos Materiais, Seção de Engenharia Mecânica e de Materiais, Instituto Militar de Engenharia, Rio de Janeiro, RJ, Brasil.. 


\section{INTRODUÇÃO}

As resinas epoxídicas são polímeros termofixos caracterizados pela presença de grupos glicidila em sua molécula. Através da reação do grupo glicidila com um agente reticulante adequado (endurecedor), uma estrutura tridimensional é formada. A epicloridrina (1-cloro-2,3 - epóxi - propano) é o agente universal portador do grupo epóxi que irá reagir com espécies químicas que tem hidrogênios ativos. $O$ bisfenol $A$ - é a espécie química mais comum que possui esses hidrogênios ativos. $\mathrm{Na}$ Figura 1 encontra-se ilustrada a reação de produção da resina epóxi mais comum, o diglicidil éter do bisfenol $A$ (DGEBA). As resinas epoxídicas podem, entre outras aplicações, ser utilizadas como adesivos, as quais são polímeros usados para unir outros polímeros, metais, cerâmicos ou compósitos, e, neste caso, organizamse em adesivos quimicamente reativos. Como este tipo de adesivo, os epóxis constituem-se em sistemas de dois componentes, os quais passam pelo processo de cura quando duas resinas são misturadas [1].

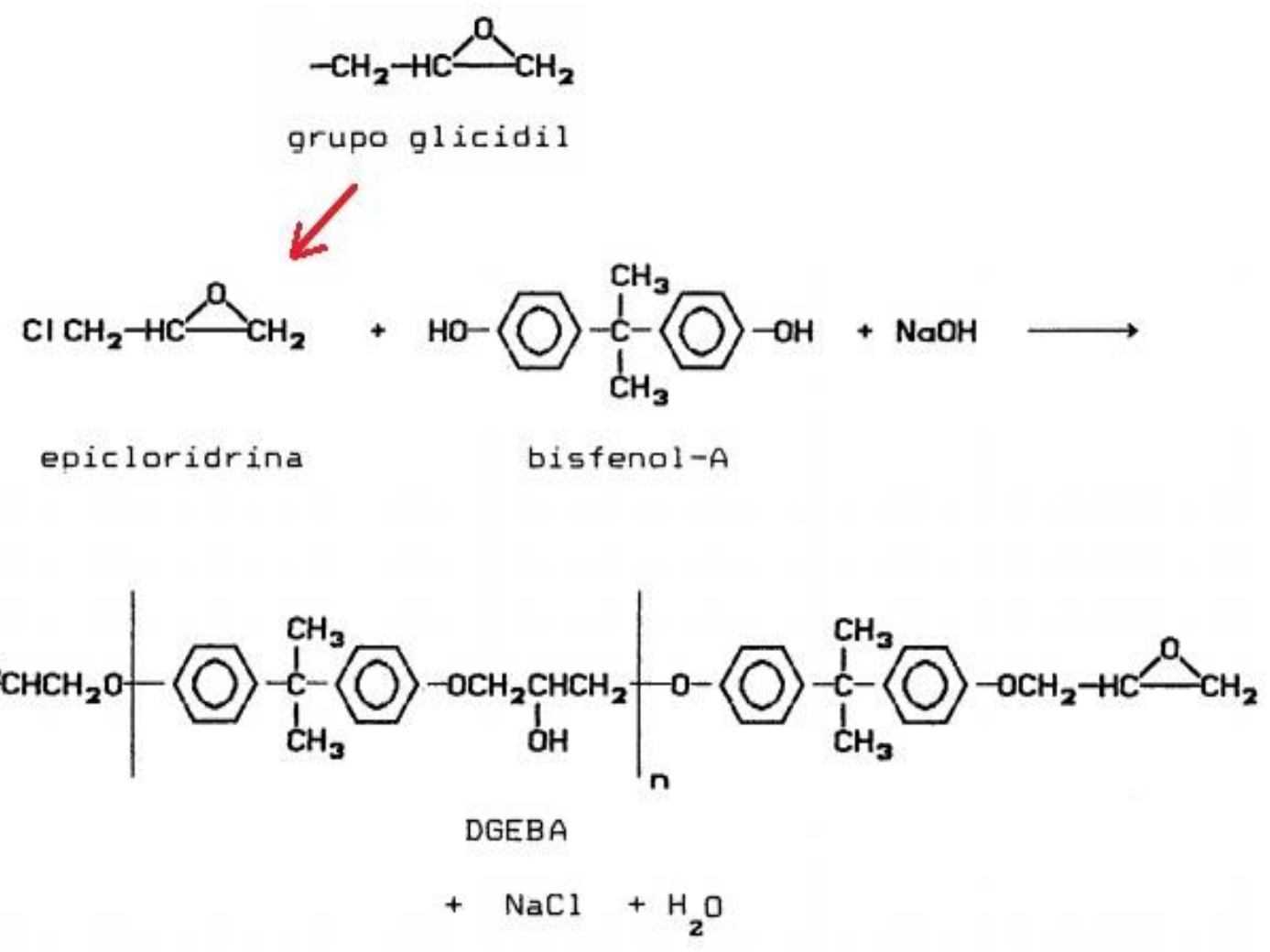

Figura 1. Reação de produção da resina epóxi [2] (adaptado).

Entre as suas características principais, pode-se citar: ótima combinação de propriedades mecânicas; boas propriedades elétricas; estáveis em termos dimensionais; adesão eficiente; excelente resistência à corrosão; e baixo custo. Entre as suas aplicações típicas, tem-se: adesivos, moldes elétricos, componentes para os setores automotivo e aeroespacial, revestimentos de proteção, artigos esportivos e matrizes para compósitos com reforço de fibras [1].

Contudo, o estudo da cinética da reação de cura das resinas epóxis, bem como dos compósitos de matriz polimérica, tem sido objeto de estudo por pesquisadores de diversas áreas [3-4]. Na produção de compósitos de matriz epoxídica reforçados por fibras naturais [5-9], após a mistura do polímero e das fibras na proporção desejada, 
o tempo de cura normalmente utilizado nos trabalhos é de 24 horas [10-13]. No entanto, este tempo é superior ao necessário para a completa reação de cura da matriz polimérica e, por conseguinte, do compósito a ser fabricado.

No presente trabalho foi realizada uma análise por calorimetria diferencial de varredura (DSC), mostrando que o tempo total de cura da resina epóxi é inferior a 24h. Além disso, há indícios que as fibras de malva utilizadas como reforço na proporção de $20 \%$ em volume, podem também reduzir o tempo total de cura do compósito de matriz polimérica. O que pode proporcionar uma maior disponibilidade de equipamentos, como prensas e moldes metálicos, assim como redução nos custos totais de produção.

\section{MATERIAIS E MÉTODOS}

Para a análise por calorimetria diferencial de varredura (DSC) as fibras de malva foram cominuídas e colocadas em cadinho de alumínio do equipamento Netzsch modelo DSC 404 F1 Pegasus, pertencente ao IME, operando em atmosfera de nitrogênio com taxas de aquecimento de $5,7,5$ e $10^{\circ} \mathrm{C} / \mathrm{min}$, em um intervalo de temperatura de 20 a $400^{\circ} \mathrm{C}$. Foram ensaiadas fibras de malva, epóxi puro e 0 compósito de epóxi reforçado com fibras de malva. O método descrito pela ASTM E698 foi utilizado para calcular os seguintes parâmetros cinéticos de cura: Energia de ativação "E" ( $\mathrm{kJ} / \mathrm{mol})$, fator pré-exponencial " $Z$ " $\left(\min ^{-1}\right)$, taxa "K" $\left(\min ^{-1}\right)$ constante para cada temperatura e o tempo médio de cura " $t_{1 / 2}$ " para o epóxi puro e o compósito reforçado por fibras de malva, em temperaturas de $25^{\circ} \mathrm{C}$ a $100^{\circ} \mathrm{C}$. A Figura 2 mostra o equipamento que foi utilizado na caracterização supracitada.

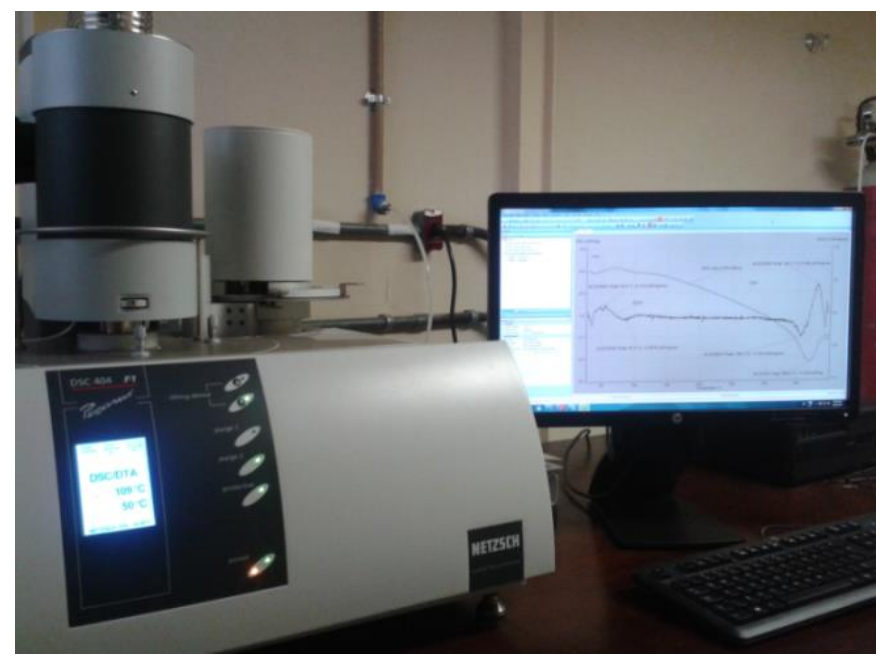

Figura 2. Equipamento Netzsch modelo DSC 404 F1 Pegasus, pertencente ao IME.

\section{RESULTADOS E DISCUSSÃO}

Foram determinados os parâmetros cinéticos de cura para a resina epóxi DGEBATETA, bem como para o compósito de epóxi reforçado com $20 \% \mathrm{v} / \mathrm{v}$ de fibras de malva, conforme o previsto na ASTM E698. As Figuras 3 e 4 mostram os gráficos de temperatura $\left({ }^{\circ} \mathrm{C}\right) \times$ fluxo de calor $(\mathrm{mW})$ para as taxas de $5,7,5$ e $10^{\circ} \mathrm{C} / \mathrm{min}$ utilizados na determinação dos parâmetros cinéticos de cura para os materiais supracitados. 


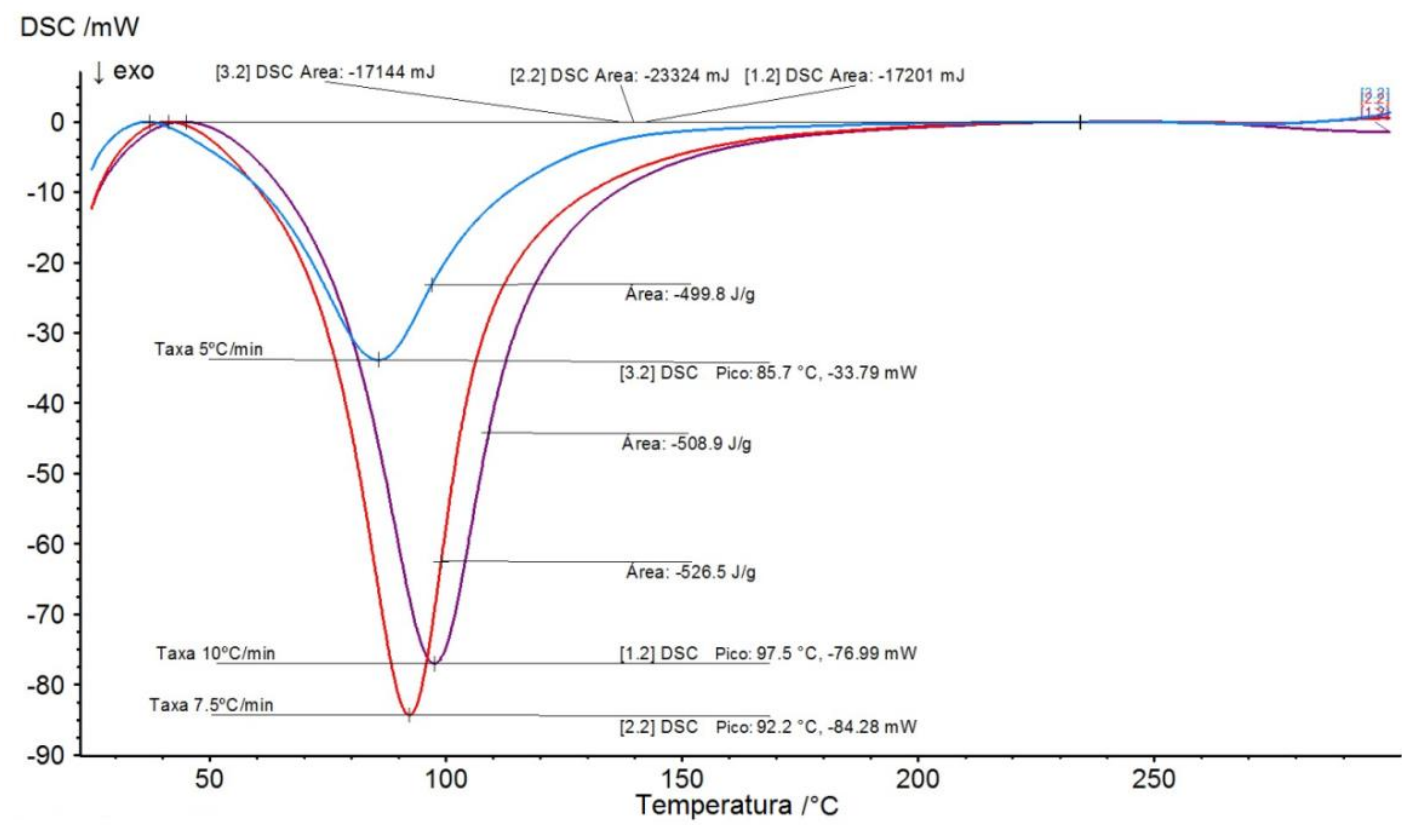

Figura 3. Curvas DSC para resina epóxi DGEBA-TETA nas taxas de aquecimento $5,7,5$ e $10^{\circ} \mathrm{C} / \mathrm{min}$ para determinação de parâmetros cinéticos de cura.

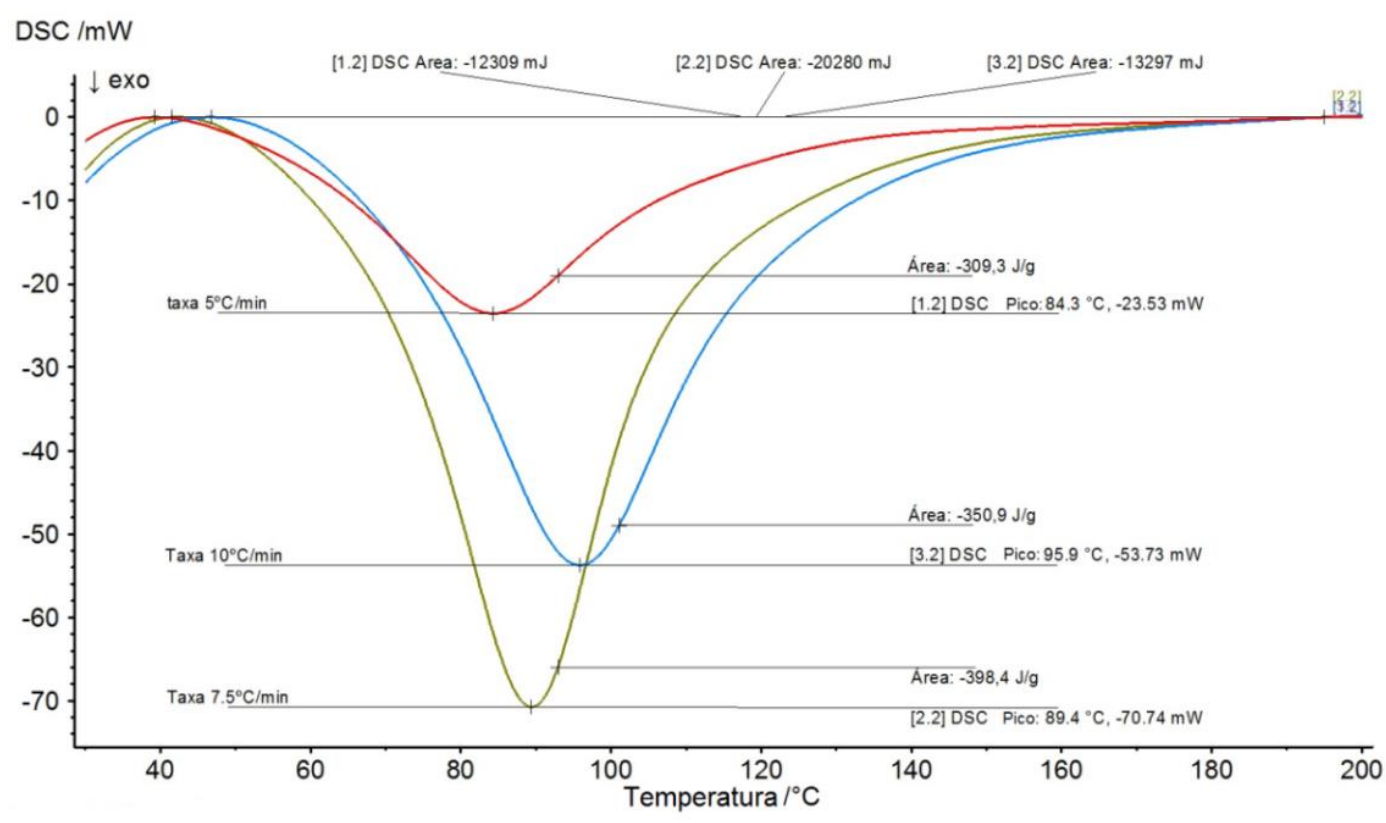

Figura 4. Curvas DSC para o compósito epóxi reforçado com $20 \% \mathrm{v} / \mathrm{v}$ de fibras de malva nas taxas de aquecimento $5,7,5$ e $10^{\circ} \mathrm{C} /$ min para determinação de parâmetros cinéticos de cura.

A Tabela 1 mostra os resultados obtidos para a energia de ativação "E" (kJ/mol), fator pré-exponencial " $\mathrm{Z}$ " $\left(\mathrm{min}^{-1}\right)$ e o tempo médio para alcançar $50 \%$ de cura " $\mathrm{t}_{1 / 2}$ " para o epóxi puro e o compósito reforçado por fibras de malva, em temperaturas variando na faixa de $25^{\circ} \mathrm{C}$ a $100^{\circ} \mathrm{C}$. 
Tabela 1. Parâmetros cinéticos de cura para resina epóxi DGEBA-TETA e compósito de epóxi reforçado com $20 \% \mathrm{v} / \mathrm{v}$ de fibras de malva.

\begin{tabular}{|c|c|c|c|c|c|}
\hline $\begin{array}{l}\text { Materiais } \\
\text { ensaiados }\end{array}$ & $\begin{array}{l}\text { Energia } \\
\text { de } \\
\text { ativação } \\
\text { (kJ/mol) }\end{array}$ & $\begin{array}{c}\text { Fator pré- } \\
\text { exponencial } \\
\text { "Z" } \\
(1 / \mathrm{min})^{*} 10^{5}\end{array}$ & $\begin{array}{c}\text { Temperaturas } \\
\left({ }^{\circ} \mathrm{C}\right)\end{array}$ & $\begin{array}{c}\text { Taxa } \\
\text { constante } \\
\text { "k" }(1 / \text { min })\end{array}$ & $\begin{array}{l}\text { Tempo } \\
\text { médio para } \\
\text { alcançar } \\
50 \% \text { de cura } \\
\text { " } \mathrm{t}_{1 / 2} \text { " (min) }\end{array}$ \\
\hline \multirow{4}{*}{$\begin{array}{l}\text { Resina epóxi } \\
\text { DGEBA-TETA }\end{array}$} & \multirow{4}{*}{58,971} & \multirow{4}{*}{1080} & 25 & 0,004997 & 138,68 \\
\hline & & & 50 & 0,031536 & 21,98 \\
\hline & & & 80 & 0,203849 & 3,4 \\
\hline & & & 100 & 0,598709 & 1,16 \\
\hline \multirow{4}{*}{$\begin{array}{l}\text { Compósito de } \\
\text { epóxi reforçado } \\
\text { com } 20 \% \text { v } / v \text { de } \\
\text { fibras de malva }\end{array}$} & \multirow{4}{*}{44,536} & \multirow{4}{*}{8,036} & 25 & 0,012540 & 55,26 \\
\hline & & & 50 & 0,050412 & 13,75 \\
\hline & & & 80 & 0,206369 & 3,36 \\
\hline & & & 100 & 0,465602 & 1,49 \\
\hline
\end{tabular}

Os resultados mostram que a medida que a temperatura cresce, em ambos os casos, o tempo médio para alcançar $50 \%$ de cura é reduzido. Este resultado já era esperado por se tratar de processos termicamente ativados, modelados pela equação de Arrhenius [14]. No entanto, comparativamente entre os materiais ensaiados, percebe-se que para as mesmas temperaturas o compósito de epóxi reforçado com fibras de malva apresentou redução no tempo médio para alcançar $50 \%$ de cura. Fato este que pode ser justificado, já que as fibras funcionam como sítios nucleadores do processo de cura do compósito [3]. No entanto, conforme a ASTM E698 e alguns autores que estudaram a cinética de cura de resinas poliméricas [3;4;15], o processo é modelado como um resfriamento homogêneo e o expoente da equação de Avrami " $n$ " foi considerado com valor unitário [16]. A Equação 1 mostra a relação completa de Avrami.

$$
1-X_{\text {tranf. }}=e^{-k t^{n}}
$$

Sendo:

$X_{\text {transf. }}=$ Fração transformada;

$\mathrm{k}=$ taxa constante para cada temperatura $\left(\mathrm{min}^{-1}\right)$;

$\mathrm{t}=$ tempo de transformação (cura) em minutos;

$\mathrm{n}=$ expoente de Avrami ( $\mathrm{n}=1$ para ASTM E698).

Com isso, foi monitorada a cura em tempo real através de DSC na temperatura fixa de $25^{\circ} \mathrm{C}$ para a resina epóxi e para o compósito reforçado com fibras de malva, com o objetivo de verificar qual o valor real do expoente da equação de Avrami. As Figuras 5 e 6 mostram as curvas obtidas. 


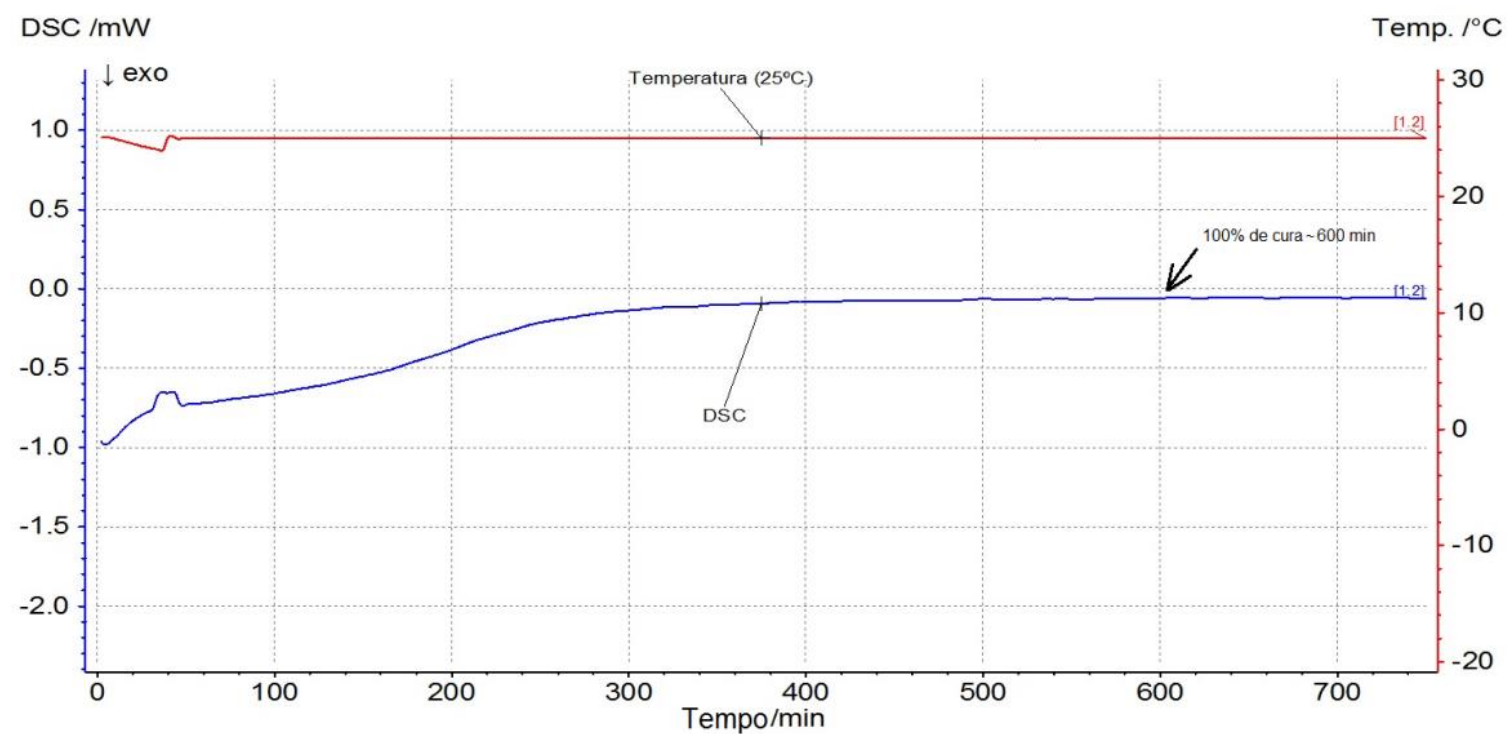

Figura 5. Curva isotérmica de DSC a $25^{\circ} \mathrm{C}$ para a resina epóxi DGEBA-TETA.

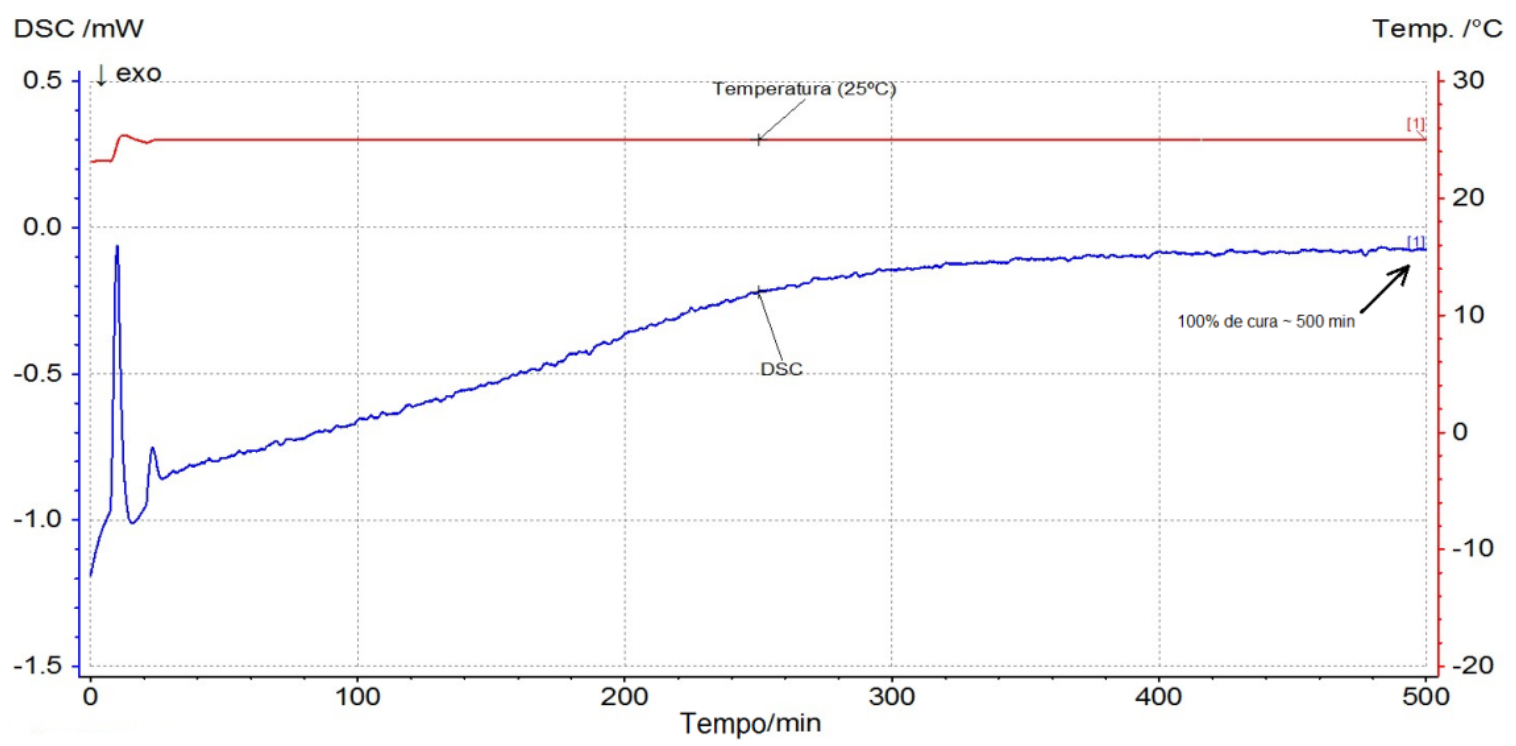

Figura 6. Curva isotérmica de DSC a $25^{\circ} \mathrm{C}$ para o compósito de epóxi reforçado com $20 \% \mathrm{v} / \mathrm{v}$ de fibras de malva.

As citadas figuras mostram um tempo real de cura a $25^{\circ} \mathrm{C}$ de 600 min para a resina epóxi e 500 min para o compósito de epóxi reforçado com fibras de malva. Com isso, recalculou-se o expoente " $n$ " na Equação 1 obtendo-se $n=0,87$ para resina epóxi DGEBA-TETA e $n=0,73$ para o compósito de epóxi reforçado com fibras de malva. A Tabela 2 mostra os valores de tempo médio para alcançar $50 \%$ de cura, recalculados a partir dos novos expoentes de Avrami. Cabe ressaltar que para as temperaturas mais elevadas $\left(>80^{\circ} \mathrm{C}\right)$ não houve diferença entre os valores de tempo previstos pelas Tabelas 1 e 2, provavelmente em função da cinética de grande velocidade envolvida no processo de cura. 
Tabela 2. Tempos médios de cura recalculados a partir dos expoentes de Avrami obtidos da curva real a $25^{\circ} \mathrm{C}$ da resina epóxi e do compósito de epóxi reforçado com $20 \% \mathrm{v} / \mathrm{v}$ de fibras de malva.

\begin{tabular}{|c|c|c|c|c|}
\hline $\begin{array}{c}\text { Materiais } \\
\text { ensaiados }\end{array}$ & $\begin{array}{c}\text { Coeficientes da } \\
\text { equação de } \\
\text { Avrami } \\
\text { (recalculados) }\end{array}$ & $\begin{array}{c}\text { Temperaturas } \\
\left({ }^{\circ} \mathrm{C}\right)\end{array}$ & $\begin{array}{c}\text { Taxa } \\
\text { constante } \\
\text { "k" (1/min) }\end{array}$ & $\begin{array}{c}\text { Tempo médio } \\
\text { para alcançar } \\
50 \% \text { de cura } \\
\text { "t } 1 / 2 " \text { (min) }\end{array}$ \\
\hline \multirow{4}{*}{$\begin{array}{l}\text { Resina epóxi } \\
\text { DGEBA-TETA }\end{array}$} & \multirow{4}{*}{0,87} & 25 & 0,004997 & 289,79 \\
\hline & & 50 & 0,031536 & 34,87 \\
\hline & & 80 & 0,203849 & 4,08 \\
\hline & & 100 & 0,598709 & 1,18 \\
\hline \multirow{4}{*}{$\begin{array}{l}\text { Compósito de } \\
\text { epóxi reforçado } \\
\text { com } 20 \% \text { v/v de } \\
\text { fibras de malva }\end{array}$} & \multirow{4}{*}{0,73} & 25 & 0,012540 & 243,72 \\
\hline & & 50 & 0,050412 & 36,24 \\
\hline & & 80 & 0,206369 & 5,26 \\
\hline & & 100 & 0,465602 & 1,72 \\
\hline
\end{tabular}

\section{CONCLUSÃo}

O estudo da cinética de cura da resina epóxi e do compósito reforçado com $20 \% \mathrm{v} / \mathrm{v}$ de fibras de malva com base na ASTM E698 determinou as energias de ativação $(58,971 \mathrm{~kJ} / \mathrm{mol}$ e $44,536 \mathrm{~kJ} / \mathrm{mol})$, os fatores pré-exponenciais " $Z$ " $\left(1,08 \times 10^{8} \mathrm{~min}^{-1} \mathrm{e}\right.$ $\left.8,04 \times 10^{5} \mathrm{~min}^{-1}\right)$ e os tempos para alcançar $50 \%$ da reação de cura $\left(\mathrm{t}_{1 / 2}\right)$ nas temperaturas de $25^{\circ} \mathrm{C}$ a $100^{\circ} \mathrm{C}$. Para a temperatura de $25^{\circ} \mathrm{C}$ foram encontrados $t_{1 / 2}$ de 138,68 e 55,26 min. No entanto, como a ASTM E698 considera a constante "n" da equação de Avrami igual a unidade, foram obtidas curvas de cura isotérmicas a $25^{\circ} \mathrm{C}$ e obtidos os tempos reais de cura. Com isso, os valores de " $n$ " foram recalculados para $n=0,87$ e $n=0,73$ para a resina epóxi e para os compósitos reforçados com $20 \% \mathrm{v} / \mathrm{v}$ de fibras de malva. Os tempos finais $\left(\mathrm{t}_{1 / 2}\right)$ a $25^{\circ} \mathrm{C}$ obtidos com os novos valores de "n" foram 289,79 e 243,72 min respectivamente.

\section{Agradecimentos}

Os autores deste trabalho agradecem ao CNPq, à FAPERJ e à CAPES por meio do PDS pelo financiamento da pesquisa, através do programa 31007015006P4.

\section{REFERÊNCIAS}

1 Askeland DR, Phulé PP. Ciência e Engenharia dos Materiais. São Paulo: Cengage Learning; 2008.

2 Rodrigues MRA. Estudo da reação de cura da resina epóxi (araldit F) com anidrido ftálico e trietilamina como iniciador [Dissertação de Mestrado]. Rio Grande do Sul: Universidade Federal do Rio Grande do Sul; 1991.

3 Costa ML, Rezende MC, Pardini LC. Métodos de estudo da cinética de cura de resinas epóxi. Polímeros. 1999;9(2):37-44.

4 Menezes GW, Monteiro SN, D'almeida JRM, Neto HSN. Análise térmica da resina epóxi DGEBA/TETA para formulações diferentes da razão estequiométrica. ABM. 2004;1(1):12-16. 
5 Mohanty AK, Misra M, Hinrichsen G. Biofibres, Biodegradable Polymers and Bio-composites: An overview. Macromolecular Materials and Engineering. 2000;276/277(1):1-24.

6 Sahed DN, Jog JP. Natural fiber polymer composites: a review. Advances in Polymer Technology. 1999;18(4):351-363.

7 Bledzki AK, Gassan J. Composites reinforced with cellulose based fibers. Progress in Polymer Science. 1999;24(2):221-274.

8 Monteiro SN, Lopes FPD, Barbosa AP, Bevitori AB, Da Silva ILA, Da Costa LL. Natural lignocellulosic fibers as engineering materials - An overview. Metallurgical and Materials Transactions A. 2011;42A:2963-2974.

9 Margem JI. Estudo das características estruturais e propriedades de compósitos poliméricos reforçados com fibras de malva [Tese de Doutorado]. Rio de Janeiro: Universidade Estadual do Norte Fluminense; 2013.

10 Luz FS. Avaliação do comportamento balístico de blindagem multicamada com compósito de epóxi reforçado com fibra de juta [Dissertação de Mestrado]. Rio de Janeiro: Instituto Militar de Engenharia; 2014.

11 Milanezi TL. Comportamento balístico da fibra de rami em blindagem multicamadas [Dissertação de Mestrado]. Rio de Janeiro: Instituto Militar de Engenharia; 2015.

12 Braga FO. Comportamento balístico de uma blindagem multicamada utilizando compósito poliéster-curauá como camada intermediária [Dissertação de Mestrado]. Rio de Janeiro: Instituto Militar de Engenharia; 2015.

13 Silva LC. Comportamento balistico do composito epoxi-curaua em blindagem multicamada [Tese de Doutorado]. Rio de Janeiro: Instituto Militar de Engenharia; 2014.

14 Callister WD, Rethwish DG. Materials Science and Engineering - An Introduction. New York, NY: John Wiley \& Sons; 2012.

15 D'Almeida JRM, Menezes GW, Monteiro SN. Ageing of the DGEBA/TETA epoxy system with off-stoichiometric compositions. 2003;6(3):415-420.

16 Avrami M. Kinetics of Phase Change. I General Theory. The Journal of Chemical Physics. 1939;7(12):1103-1112. 